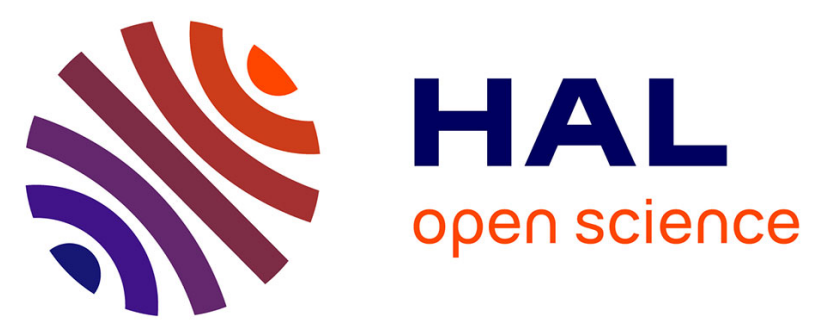

\title{
Action, vis à vis de quelques agents parasitaires, de deux fractions élicitrices issues de Phytophthora capsici appliquées sur organes en survie et plantules de diverses espèces végétales
}

\author{
Paul-Michel Molot, Pierre Mas, Hervé Lecoq, Georges Marchoux
}

\section{To cite this version:}

Paul-Michel Molot, Pierre Mas, Hervé Lecoq, Georges Marchoux. Action, vis à vis de quelques agents parasitaires, de deux fractions élicitrices issues de Phytophthora capsici appliquées sur organes en survie et plantules de diverses espèces végétales. Agronomie, 1984, 4 (9), pp.835-842. 10.1051/agro:19840905 . hal-02728595

\section{HAL Id: hal-02728595 \\ https://hal.inrae.fr/hal-02728595}

Submitted on 2 Jun 2020

HAL is a multi-disciplinary open access archive for the deposit and dissemination of scientific research documents, whether they are published or not. The documents may come from teaching and research institutions in France or abroad, or from public or private research centers.
L'archive ouverte pluridisciplinaire HAL, est destinée au dépôt et à la diffusion de documents scientifiques de niveau recherche, publiés ou non, émanant des établissements d'enseignement et de recherche français ou étrangers, des laboratoires publics ou privés. 


\section{Action, vis-à-vis de quelques agents parasitai- res, de deux fractions élicitrices issues de Phytophthora capsici appliquées sur organes en survie et plantules de diverses espèces végétales}

Paul-Michel MOLOT, Pierre MAS, Hervé LECOQ \& Georges MARCHOUX

I.N.R.A., Station de Pathologie végétale, Centre de recherches d'Avignon, B.P. 94, F 84140 Montfavet

Deux fractions élicitrices, issues de Phytophthora capsici et partiellement purifiées, G4 et G5 sont appliquées sur organes foliaires en survie de Capsicum annuum et de Cucumis melo. Elles ont pour effet non seulement de réduire l'infection fongique par $P$. capsici (tabl. 1) ou Colletotrichum lagenarium (tabl. 2), mais encore d'augmenter de façon significative le nombre de lésions locales produites par le virus de la mosaïque du concombre (tabl. 1) ou celui de la criblure du melon (tabl. 2).

Sur cotylédons de $C$. annuum, Lycopersicon esculentum et $C$. melo en survie, la fraction G4 est très efficace si elle est déposée au site de contamination ou appliquée sur une blessure (tabl. 3). On observe alors une protection du piment, de la tomate et du melon vis-à-vis de $P$. capsici, $P$. infestans et $C$. lagenarium (tabl. 4) et une augmentation du nombre de lésions locales virales (tabl. 5).

Le caractère systémique et non spécifique de la protection est discuté.

Mots clés additionnels : Colletotrichum, virus de la mosaique du concombre souche $N$, virus de la criblure du melon, Capsicum annuum, Lycopersicon esculentum, Cucumis melo, Vigna unguiculata, Nicotiana tabacum, blessure, protection systémique, induction de résistance, hypersensibilité.

Two partially purified elicitor fractions G4 and G5 obtained from Phytophthora capsici and applied on detached leaves or cotyledons of Capsicum annuum or Cucumis melo reduced infection by $P$. capsici (table 1) and Colletotrichum lagenarium (table 2) respectively. They also induced a significant increase in the number of local lesions produced by cucumber mosaic virus (CMV-N strain) on pepper (table 1) and by muskmelon necrotic spot virus (MNSV-E8 strain) on melon (table 2).

The G4 elicitor fraction was very efficient on detached cotyledons of C. annuum, Lycopersicon esculentum and C. melo when applied at the site of pathogen inoculation. In order to be efficient when applied to a determined site, it must be deposited into a wound (table 3 ); when the G4 elicitor fraction was applied to plantlets in this way, protection was observed against $P$. capsici, $P$. infestans and $C$. lagenarium in pepper, tomato and melon respectively (table 4). A significant increase in local lesion numbers produced by CMV-N strain on pepper, tobacco and Vigna unguiculata and by MNSV-E8 on melon was also observed under these conditions (table 5).

The non specific and systemic aspect of the effect of the elicitor fraction is discussed.

Additional key words : Phytophthora capsici, Colletotrichum lagenarium, cucumber mosaic virus (CMV-N strain), muskmelon necrotic spot virus (MNSV-E8 strain), Capsicum annuum, Lycopersicon esculentum, Cucumis melo, Vigna unguiculata, Nicotiana tabacum, wound, systemic protection, induced resistance, hypersensitivity. 


\section{INTRODUCTION}

Dans une précédente publication (MOLOT et al., $1980 b$ ), nous avons signalé avoir obtenu, à partir du filtrat de culture de Phytophthora capsici Leon., 2 fractions G4 et G5 susceptibles d'induire une réaction de défense. Expérimentées sur organes foliaires de piment en survie, ces fractions ont donné des résultats positifs lorsque :

- les organes foliaires flottent sur la fraction élicitrice et sont contaminés en un point quelconque du limbe (COULOMB et al., 1980) ;

- les organes foliaires, flottant sur l'eau, reçoivent une microgoutte d'éliciteur et sont contaminés en ce même endroit (MOLOT et al., 1980b ; MOLOT et al., 1983).

Afin de donner à ces résultats un caractère plus général, les fractions G4 et G5 ont été éprouvées dans le présent travail sur d'autres plantes que le piment et vis-à-vis d'autres parasites (champignons ou virus) que P. capsici.

Nous nous sommes proposé aussi de vérifier le caractère systémique de l'induction, en pratiquant l'élicitation et la contamination en des sites différents sur tissu intact ou blessé. Enfin, nous avons précisé sur plantule en place, l'effet d'une élicitation préalable sur l'évolution d'une infection parasitaire.

\section{MATÉRIEL ET MÉTHODES}

\section{A. Matériel végétal et parasitaire}

Deux variétés de piment (Capsicum annuum L.), l'une sensible à $P$. capsici, "Yolo Wonder », l'autre résistante "Phyo 636 », ont été choisies, ainsi que la tomate (Lycopersicon esculentum Mill.) « Monalbo », le melon (Cucumis melo L.) «Doublon ", Vigna unguiculata (L.) Walp. var. "Black» et Nicotiana tabacum L. var. "Xanthi n. c. ".

Ce matériel, produit en serre, est utilisé au stade cotylédons- 2 feuilles; exceptionnellement, on a fait usage de feuilles adultes de piment (5 et $6^{\mathrm{e}}$ étages) prélevées sur plantes à l'apparition des boutons floraux.

\section{Agents cryptogamiques}

Pour contaminer le piment, la tomate et le melon, nous utilisons respectivement la souche 15 de $P$. capsici isolée du piment et cultivée sur milieu V8, la souche 855 de Phytophthora infestans (Mont.) de By isolée de la tomate et cultivée sur milieu avoine-pois chiche et un isolat de Colletotrichum lagenarium (Pass.) Ell. \& Halst. isolé de melon par le Centre de Physiologie végétale de Toulouse et cultivé sur milieu avoine.

\section{Virus}

Le virus de la mosaïque du concombre (CMV) souche $\mathrm{N}$ a été cloné à partir de l'isolat de TROUTMAN \& FULTON (1958). Cette souche présente la particularité de produire des lésions locales nécrotiques sur les différents hôtes étudiés (MARCHOUX, 1975).

La souche (E8) du virus de la criblure du melon utilisée pour contaminer cette plante présente les mêmes propriétés que celles décrites par MARROU \& RISSER
(1967) et, en particulier, elle induit des lésions locales chez de nombreuses variétés de melon. Cependant, elle ne provoque pas de lésions locales chez Chenopodium quinoa et Chenopodium amaranticolor. Elle est par ailleurs, selon LECOQ (travaux non publiés), identique à celle du muskmelon necrotic spot virus (MNSV) décrit par GONZALES-GARZA et al. (1979).

\section{B. Mise en contact du végétal avec la fraction élicitrice}

Nous utilisons 2 fractions inductrices de résistance obtenues à partir du filtrat de culture de la souche 15 de $P$. capsici. Celui-ci est concentré et précipité à l'éthanol et les surnageants dialysés. La partie non dialysable comporte 2 sous-unités, l'une insoluble dans l'eau G4, l'autre soluble G5 (MOLOT et al., $1980 b$ ). La fraction G4 est employée exclusivement pour les dépôts localisés en microgouttes; par ailleurs, à la surface d'une solution de la fraction G5 ( $20 \mathrm{ml}$ par boîte de Petri), on place en survie des organes foliaires. La concentration pour G4 et G5 est toujours de $0,5 \mathrm{mg} / \mathrm{ml}$ d'eau. Les opérations peuvent être menées soit sur organes en survie soit sur plantules.

\section{Dépôt d'une microgoutte de la fraction G4 sur organes en survie}

L'organe foliaire « flotte » sur l'eau. On dépose, en principe au centre du limbe, $25 \mu \mathrm{l}$ de G4 ; dans certains cas, en particulier pour étudier l'effet d'élicitation à distance, on décale le dépôt vers les extrémités distales ou proximales du limbe (voir tabl. 3). $24 \mathrm{~h}$ après, quand la goutte est résorbée, on procède à la contamination fongique.

\section{Mise en survie d'organes foliaires d̀ la surface d'une solution de la fraction 65}

Cette opération se déroule aussitôt après la contamination (voir plus loin). Les organes sont prélevés, mis en service sur G5 par la face inférieure pendant $24 \mathrm{~h}$, puis transférés sur l'eau.

\section{Dépôt d'une microgoutte de la fraction G4 sur plantules}

Avec du papier de verre, on blesse légèrement l'extrémité distale d'un cotylédon et l'on dépose à cet endroit, sur la face supérieure du limbe, $50 \mu \mathrm{l}$ de G4. La goutte est recouverte d'un petit carré de parafilm $(15 \times 15 \mathrm{~mm})$. La contamination virale ou fongique est réalisée $48 \mathrm{~h}$ après, selon la méthode décrite cidessous.

\section{Méthodes de contamination}

\section{Inoculations fongiques}

Chaque traitement comporte 20 organes en survie ou 10 plantules.

\section{a) Sur organes en survie}

La contamination est réalisée, simultanément ou $24 \mathrm{~h}$ après l'élicitation, de façon ponctuelle, soit par mycélium, soit par dépôt de spores :

- La contamination par mycélium est réservée aux feuilles adultes de piment. Celles-ci, après avoir été blessées au centre du limbe à l'aide d'une aiguille lancéolée, sont infectées par un fragment calibré $(\varnothing=4 \mathrm{~mm})$ d'une culture mycélienne de $P$. capsici. 


\section{b) Sur cotylédons ou jeunes feuilles}

L'apport d'inoculum est effectué sous forme d'une goutte de $10 \mu \mathrm{l}$, déposée en principe au centre du limbe et au même endroit que l'éliciteur (si celui-ci a été mis sous forme de goutte). Pour étudier l'effet d'induction à distance (voir tabl. 3), la contamination est cependant réalisée en un point proche du pétiole et ne correspondant pas forcément au site de dépôt de l'éliciteur.

Les concentrations en inoculum sont :

- $7.10^{4}$ zoospores $/ \mathrm{ml}$ pour $P$. capsici.

- 2,5.10 $0^{3}$ sporanges $/ \mathrm{ml}$ pour $P$. infestans.

$-1.10^{4}$ spores $/ \mathrm{ml}$ pour C. lagenarium.

\section{c) Sur plantules}

On contamine, $48 \mathrm{~h}$ après l'élicitation, par pulvérisation de spores. L'inoculum de $P$. capsici renferme $5.10^{3}$ ou $1.10^{4}$ zoospores $/ \mathrm{ml}$ pour «Yolo Wonder » et des quantités 10 fois supérieures pour la variété résistante «Phyo 636 »; les jeunes tomates sont pulvérisées avec 750 sporanges de $P$. infestans $/ \mathrm{ml}$ et les melons avec $1.10^{3}$ spores de $C$. lagenarium $/ \mathrm{ml}$. Le matériel est ensuite maintenu en chambre humide pendant $3 \mathrm{j}$ à $20^{\circ} \mathrm{C}$.

\section{Inoculations virales}

Juste avant leur mise en survie, les organes foliaires sont contaminés, par inoculation mécanique de la surface entière du limbe, selon la méthode couramment utilisée au laboratoire (MARROU, 1967).

Dans le cas de plantules, la même opération est réalisée sur le cotylédon opposé à celui qui a été élicité ou sur la feuille supérieure.

\section{Notations}

\section{Attaques fongiques}

Sur feuilles adultes de piment, on mesure, $3 \mathrm{j}$ après la contamination, le diamètre de la zone nécrosée envahie par $P$. capsici.

Sur jeunes organes excisés, qu'il s'agisse de $P$. capsici ou de $C$. lagenarium, les notations sont effectuées selon 3 classes définies par ailleurs (MOLOT et al., 1980b) :

note 0 : aucun symptôme,

note 1 : présence de petits points nécrotiques sans évolution ultérieure,

note 3 : apparition d'une nécrose gagnant l'ensemble de l'organe foliaire.

Les notes cumulées sont exprimées en pourcentage, 100 p. 100 correspondant à la sensibilité maximum.

Sur plantules, les contrôles sont réalisés quotidiennement en affectant chaque plante d'une note de sensibilité allant de 0 à 5 . Le résultat final est exprimé en pourcentage $(0$ p. $100=$ résistant ; 100 p. $100=$ mort $)$.

\section{Attaques virales}

La notation consiste en un dénombrement des lésions locales nécrotiques. L'interprétation statistique des résultats est effectuée au moyen du test $t$ de STUDENT.

\section{RÉSULTATS}

\section{A. Réponse des organes foliaires en survie sur G5 à une contamination virale ou fongique}

Chez les feuilles adultes de piment en survie sur la fraction élicitrice, on observe une augmentation du nombre de lésions dues à la souche $\mathrm{N}$ de $\mathrm{CMV}$ et à une diminution du diamètre de la zone de tissu nécrosée par $P$. capsici (tabl. 1). Ce phénomène semble d'ailleurs amplifié chez la variété résistante.

\section{TABLEAU 1}

Réponse des feuilles adultes de 2 variétés de piment en survie sur l'eau ou sur la fraction élicitrice G5-15 à une contamination virale ( $C M V-N)$ ou fongique (Phytophthora capsici).

Response of detached pepper leaves maintained on water or G5-15 elicitor to an inoculation with $C M V-N$ or with Phytophthora capsici.

\begin{tabular}{|c|c|c|c|c|}
\hline \multirow[b]{3}{*}{ Témoin eau } & \multicolumn{2}{|c|}{ Yolo wonder } & \multicolumn{2}{|c|}{ Phyo 636} \\
\hline & A & $\mathbf{B}$ & $\mathbf{A}$ & $\mathbf{B}$ \\
\hline & $18,6 \pm 4,2$ & $32,4 \pm 1,1$ & $19,7 \pm 3,0$ & $14,8 \pm 1,9$ \\
\hline \multirow[t]{2}{*}{$\begin{array}{c}\text { G5-15 } \\
(0,5 \mathrm{mg} / \mathrm{ml})\end{array}$} & 28,3 & 30,9 & 35,1 & 10,5 \\
\hline & $S$ & NS & $S$ & $\mathrm{~S}$ \\
\hline
\end{tabular}

$A=$ nombre de lésions locales par feuille après infection par le CMV-N.

Mean number of local lesions per leaf.

$\mathrm{B}=$ diamètre en $\mathrm{mm}$ de la zone de tissu nécrosée par $P$. capsici.

Diameter $(\mathrm{mm})$ of leaf necrosis induced by $P$. capsici.

Lecture 3 jours après la contamination.

Measurements made 3 days after inoculation.

NS = effet non significatif.

$\mathrm{S}=$ effet significatif au seuil de 0,01 .

Une expérience identique réalisée avec des feuilles et des cotylédons de melon permet d'obtenir des résultats similaires : les tissus « élicités » par la fraction G5 présentent un plus grand nombre de lésions locales dues au virus de la criblure et une sensibilité plus faible à C. lagenarium (tabl. 2).

Les réponses obtenues par mise en survie sur la fraction G5 d'organes foliaires détachés laissent supposer un effet systémique de l'éliciteur. Nous avons donc cherché à préciser ce point.

\section{B. Efficacité de G4 appliqué sur cotylédons en survie ou sur plantules vis-à-vis d'une contamination virale ou fongique}

\section{Sur cotylédons en survie et en fonction de la loca- lisation des sites de dépôt}

Sur cotylédons bien développés de piment, tomate et melon, les contaminations artificielles par dépôt de spores ont toujours été réalisées au même endroit, c'est-à-dire plus près du pétiole que de l'extrémité distale du limbe. Seul le site de dépôt de la fraction G4 varie : il peut être soit identique au site de contamination, soit éloigné de celui-ci, en direction de l'extrémité distale du limbe d'environ $20 \mathrm{~mm}$ (tabl. 3). 
TABLEAU 2

Réponse des organes foliaires de jeunes melons « Doublon 》 (stade cotylédons-l feuille) en survie sur l'eau ou sur la fraction élicitrice G5-15 à une contamination virale (virus de la criblure) ou fongique (Colletotrichum lagenarium).

Response of detached melon (Doublon) leaves or cotyledons on water or G5-15 elicitor to inoculation with MNSV or Colletotrichum lagenarium.

\begin{tabular}{|c|c|c|c|c|}
\hline \multirow[b]{3}{*}{ Témoin eau } & \multicolumn{2}{|c|}{ Cotylédons } & \multicolumn{2}{|c|}{ Feuilles } \\
\hline & A & B & A & B \\
\hline & $18,5 \pm 3,6$ & $78 \pm 5,0$ & $11,7 \pm 4,7$ & $41 \pm 6,1$ \\
\hline \multirow[t]{2}{*}{$\begin{array}{c}\mathrm{G} 5-15 \\
(0,5 \mathrm{mg} / \mathrm{ml})\end{array}$} & 27,1 & 51,0 & 21,4 & 29,0 \\
\hline & S & & ; & NS \\
\hline
\end{tabular}

$A=$ nombre de lésions locales par organe foliaire après infection par le virus de la criblure.

Mean number of local lesions per foliar organ.

$\mathbf{B}=$ sensibilité à $C$. lagenarium exprimé en $\%$.

Susceptibility (\%) to C. lagenarium.

Lecture $7 \mathrm{j}$ après la contamination pour $C$. lagenarium,

$3 \mathrm{j}$ après la contamination pour le virus de la criblure.

Measurements made 7 days after $C$. lagenarium inoculation and

3 days after MNSV inoculation.

NS effet non significatif.

S effet significatif au seuil de 0,01 .
S'il n'y a pas blessure du limbe, l'effet de protection n'est observé que lorsque la contamination est réalisée au site du dépôt de l'éliciteur (d). En revanche, si élicitation et contamination ne se font pas au même endroit (c), on ne met en évidence aucune induction de résistance.

S'il y a blessure du limbe, l'effet obtenu sera différent selon qu'en ce lieu seront pratiquées élicitation et contamination (b) ou simplement élicitation (a). Dans le $1^{\text {er }}$ cas (b), la protection comparée à ce qui se passe en (d), est faible, vraisemblablement parce que la blessure favorise la contamination ; dans le $2^{\mathrm{e}}$ cas (a), elle est encore plus réduite, mais cependant significativement plus efficace qu'en (c). En outre, si l'on compare les témoins eau (a) et (c), il est intéressant de signaler que le fait de blesser l'extrémité distale du limbe déclenche une réaction de défense chez le végétal.

Pour obtenir un effet de protection, l'éliciteur doit donc être appliqué au site de contamination, sinon il faut le déposer sur une blessure. Ce résultat obtenu avec 3 couples hôte-parasite différents nous a incités à poursuivre notre travail, non plus sur organes en survie, mais sur plantules racinées en place.

\section{Sur plantules}

Nous apportons la fraction G4 sur une blessure pratiquée à l'extrémité du limbe (cotylédons ou feuilles)

TABLEAU 3

Efficacité de la fraction G4-15 sur 3 couples plante-parasite en fonction de son dépôt par rapport au site de contamination. Efficiency of G4-15 elicitor on three host-pathogen systems according to the sites of elicitation and inoculation.

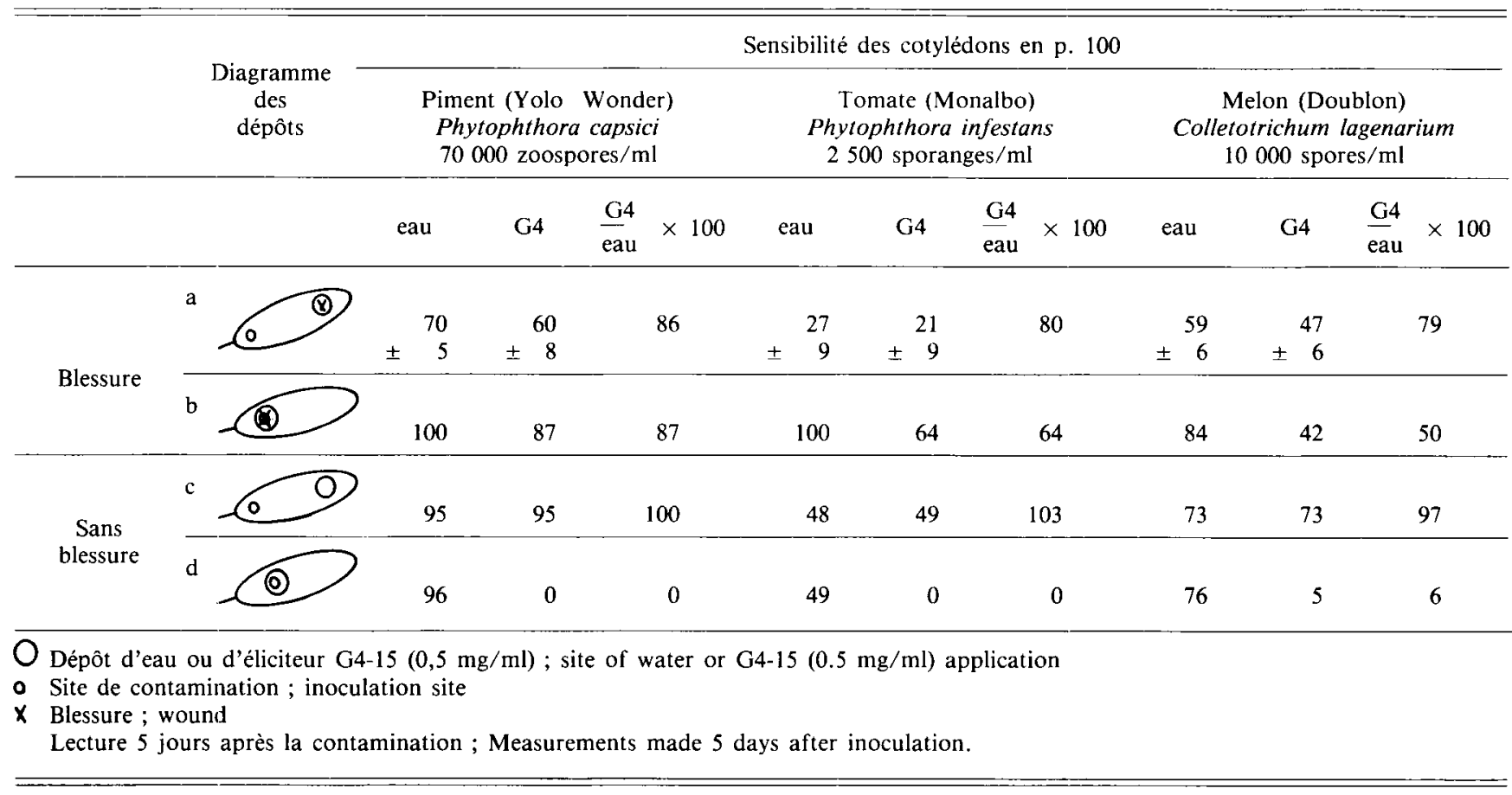

et réalisons $48 \mathrm{~h}$ après en un site différent une contamination fongique ou virale.

\section{a) Contaminations fongiques}

Le traitement par la fraction G4 entraîne une diminution de la sensibilité des 3 plantes-hôtes contami- nées (tabl. 4). Cette protection notée au $7^{\mathrm{e}} \mathrm{j}$ peut se maintenir $14 \mathrm{j}$ (et même au-delà) après la contamination. Elle est particulièrement nette dans le cas de la tomate et de $P$. infestans. En ce qui concerne le piment, on remarquera l'influence de l'inoculum sur l'extériorisation des symptômes ; c'est aux concentra- 
TABLEAU 4

Pourcentage de sensibilité du piment, de la tomate et du melon vis-à-vis de $\mathrm{P}$. capsici, P. infestans et $\mathrm{C}$. lagenarium après dépôt de l'éliciteur sur blessure d'un cotylédon de plantule. Contamination par pulvérisation.

Susceptibility (in \%) of pepper, tomato and melon to P. capsici, P. infestans and C. lagenarium after the deposit of elicitor on cotyledon wound.

\begin{tabular}{|c|c|c|c|c|c|c|c|}
\hline Parasite & \multicolumn{4}{|c|}{ Phytophthora capsici } & & $\begin{array}{l}\text { Phytophthora } \\
\text { infestans }\end{array}$ & $\begin{array}{c}\text { Colletotrichum } \\
\text { lagenarium }\end{array}$ \\
\hline Plante & \multicolumn{4}{|c|}{ Piment } & & Tomate & Melon \\
\hline Variété & \multicolumn{2}{|c|}{ Yolo Wonder } & \multicolumn{2}{|c|}{ Phyo 636} & & Monalbo & Doublon \\
\hline $\begin{array}{l}\text { Concentration } \\
\text { de l'inoculum } \\
\text { en spores/ml }\end{array}$ & $\begin{array}{c}5000 \\
\text { (zoospores) }\end{array}$ & $\begin{array}{c}10000 \\
\text { (zoospores) }\end{array}$ & $\begin{array}{c}50000 \\
\text { (zoospores) }\end{array}$ & $\begin{array}{c}100000 \\
\text { (zoospores) }\end{array}$ & & $\begin{array}{c}750 \\
\text { (sporanges) }\end{array}$ & $\begin{array}{c}1000 \\
\text { (spores) }\end{array}$ \\
\hline Témoin eau & 48 & 68 & 26 & 40 & & 70 & 97 \\
\hline \multirow[t]{2}{*}{$\begin{array}{c}\mathrm{G} 4-15 \\
(0,5 \mathrm{mg} / \mathrm{ml})\end{array}$} & 16 & 60 & 0 & 28 & & 31 & 69 \\
\hline & \multicolumn{2}{|c|}{ S } & \multicolumn{2}{|c|}{$\mathrm{S}$} & S & \multicolumn{2}{|c|}{$\mathrm{S}$} \\
\hline$\frac{\mathrm{G} 4}{\mathrm{eau}} \times 100$ & 33 & 88 & 0 & 70 & & 44 & 71 \\
\hline
\end{tabular}

Lecture 7 jours après la contamination; inoculation by spraying ; measurements made 7 days after inoculation.

NS effet non significatif.

$\mathrm{S}$ effet significatif au seuil de 0,01 .

tions en zoospores les plus faibles que l'effet de protection se manifeste le mieux. En dépit d'une pression d'inoculum plus forte, la variété résistante «Phyo 636 » révèle une capacité d'induction plus élevée que celle de «Yolo Wonder ».

\section{b) Contaminations virales}

Elles ont été réalisées par voie mécanique, sur l'organe foliaire opposé à l'organe élicité et maintenu sur la plante.

Dans tous les couples étudiés, aussi bien avec le CMV souche $\mathrm{N}$ qu'avec le virus de la criblure, on constate une augmentation significative du nombre de lésions locales chez les plantes élicitées, spécialement dans le cas du tabac (tabl. 5).

Qu'il s'agisse d'une contamination fongique ou virale, il y a, dans les 2 cas, élicitation systémique de mécanismes de protection ou de réactivité.

\section{DISCUSSION-CONCLUSION}

Les travaux relatés ici permettent de retrouver plusieurs résultats signalés par ailleurs et de présenter de nouvelles acquisitions.

Chez le piment, l'induction de résistance à $P$. capsici (tabl. 4) est non seulement plus nette chez la variété résistante «Phyo 636 » que chez la variété sensible "Yolo Wonder", mais est aussi fonction de la dose d'inoculum comme cela a déjà été observé précédemment (MOLOT et al., 1980a ; MOLOT et al., 1983).

Si l'on pratique la contamination au site même du dépôt de l'éliciteur l'effet de protection s'exerce avec le maximum d'efficacité, qu'il s'agisse de fractions membranaires (COULOMB et al., 1980b) ou de fractions issues du filtrat de culture de $P$. capsici selon le

\section{TABLEAU 5}

Influence de la fraction G4-15 appliquée à une plantule en place, sur le nombre de lésions locales par feuille, après inoculation mécanique de virus.

Effect of G4-15 elicitor applied to intact young plantlets on the number of local lesions per leaf after inoculation with virus.

\begin{tabular}{|c|c|c|c|c|}
\hline Virus & & CMV-N & & $\begin{array}{c}\text { Virus } \\
\text { de la criblure } \\
\text { MNSV }=E 8\end{array}$ \\
\hline Plante & $\begin{array}{c}\text { Piment } \\
\text { (Phyo 636) }\end{array}$ & $\begin{array}{c}\text { Vigna } \\
\text { (Black) }\end{array}$ & $\begin{array}{c}\text { Tabac } \\
\text { (Xanthi) }\end{array}$ & $\begin{array}{c}\text { Melon } \\
\text { (Doublon) }\end{array}$ \\
\hline Eau & $14 \pm 3$ & $56 \pm 10$ & $134 \pm 17$ & $37 \pm 4$ \\
\hline \multirow[t]{2}{*}{$\begin{array}{c}\mathrm{G} 4-15 \\
(0,5 \mathrm{mg} / \mathrm{ml})\end{array}$} & \multirow[t]{2}{*}{22} & 77 & 270 & 48 \\
\hline & & $s$ & & $\mathrm{~S}$ \\
\hline$\frac{\mathrm{G} 4}{\text { eau }} \times 100$ & 157 & 137 & 201 & 129 \\
\hline
\end{tabular}

Lecture 3 jours après la contamination.

Measurements made 7 days after inoculation.

$\mathrm{S}$ : effet significatif au seuil de 0,01 .

mode de fractionnement G (MOLOT et al., 1980b; MOLOT et al., 1983). En revanche, la fraction G4 appliquée sans blessure en un site différent du site de contamination se révèle sans efficacité (MOLOT et al., $1980 \mathrm{~b}$ ). Il ne faudrait cependant pas conclure hâtivement à l'absence de protection systémique, car, en laissant en survie les organes foliaires sur des préparations élicitrices, il est possible d'observer sur la face opposée du limbe une diminution de la sensibilité. Dans ces conditions expérimentales, si l'éliciteur est 
au contact de la face inférieure du limbe, on peut contaminer n'importe quel site de la face supérieure (COUlOMB et al., 1980a ; MOLOT et al., 1980a). Ici, en utilisant la fraction soluble G5 (et non pas G4), nous avons obtenu un effet protecteur sur piment et melon vis-à-vis de $P$. capsici et de $C$. lagenarium. On peut donc penser à un transport de l'éliciteur ou de son signal à travers le tissu palissadique du limbe.

Par ailleurs, cette étude a permis, dans le cas particulier des éliciteurs de $P$. capsici, de mettre en évidence des phénomènes nouveaux : le caractère non spécifique et systémique de l'induction (liée aux blessures opérées sur l'organe foliaire), l'augmentation du nombre des lésions locales virales, les différentes potentialités d'action des fractions élicitrices issues du filtrat de culture et la persistance temporaire des réactions physiologiques de défense de la plante.

L'induction par des éliciteurs d'une protection non spécifique est un phénomène bien connu, signalé chez différents modèles : tabac - Nocardia asteroides (GIANINAZZI \& MARTIN, 1975), haricot - Colletotrichum lindemuthianum (ANDERSON-PROUTY \& ALBERSHEIM, 1975), soja - Phytophthora megasperma f. sp. sojae (AYERS et al., 1976), pomme de terre - Phytophthora infestans (DOKE et al., 1979). Un éliciteur donné peut induire chez différentes plantes autant de phytoalexines différentes (CLINE et al., 1978). Les fractions G4 ou G5 extraites de $P$. capsici sont capables de susciter une réaction de défense chez des plantes très diverses (piment, melon, tomate, tabac, vigna), vis-à-vis de parasites très différents ( $P h y$ tophthora, Colletotrichum, virus).

Toutefois, ce caractère de non-spécificité, largement admis à l'heure actuelle, n'est peut-être pas aussi absolu. Les éliciteurs pourraient agir comme des lectines et être à la base de phénomènes de reconnaissance (GARAS \& KUĆ, 1981). Il convient de les produire non pas in vitro sur un milieu de culture artificielle, mais in vivo, la confrontation champignon-hôte étant nécessaire pour que la spécificité s'exprime (DE WIT \& ROSEBOOM, 1980 ; DE WIT \& SPIKMAN, 1982). De plus, parmi les glucides isolés des parois mycéliennes de Phytophthora megasperma f. sp. glycinea, les glucomannanes extraits par action d'une endo-glucanase $\beta 1-3$ du soja possèdent une activité élicitrice spécifique d'une race physiologique (KEEN et al., 1983). Nousmêmes, dans un travail non publié, avons montré qu'en fonction de leur degré de purification, certaines fractions élicitrices de Phytophthora nicotianae parasitica, actives au départ sur plusieurs plantes, présentaient une nette spécificité : toujours efficaces sur œillet vis-à-vis de ce même parasite, elles ne le sont plus sur piment vis-à-vis de $P$. capsici.

Le caractère systémique de la protection mis en évidence dans le cas d'une infection localisée préalable (JENNS et al., 1979 ; STAUB \& KUĆ, 1980 ; HAMILTON, 1980 ; ROBERTS, 1983) et aussi après injection de différents métabolites (GIANINAZZI \& MARTIN, 1975 ; ROSSIGNOL, 1979 ; TOUZÉ \& ROSSIGNOL, 1980) est également démontré ici. En effet, la fraction insoluble G4, appliquée localement sur cotylédons confère aux plantules un meilleur niveau de résistance. Cependant, cet effet n'est obtenu que s'il y a blessure au site d'élicitation.

Ajoutons que, même en l'absence d'élicitation, le simple fait de blesser entraîne une légère augmentation de résistance. La réaction des plantes aux traumatismes est un phénomène connu (GIANINAZZI, 1982). Dans le cas particulier des morsures d'insectes, il s'explique par l'apparition d'une hormone végétale de défense (P.I.I.F. = proteinase inhibitor inducing factor) qui, après migration rapide, déclenche l'accumulation d'inhibiteurs et suscite une réaction de défense généralisée (GREEN \& RYAN, 1972 ; RYAN, 1978). En présence de G4, l'effet de protection dû à la blessure est renforcé. A une meilleure pénétration de cette fraction insoluble pourrait s'ajouter le fait que certains éliciteurs ont la particularité de stimuler, chez la plante, la synthèse d'inhibiteurs protéasiques (ROBY, 1981).

Un autre point retiendra notre attention. On observe en effet qu'un traitement préalable par les fractions G4 ou G5 entraîne une augmentation du nombre de lésions locales produites dans les 4 couples virus-hôtes étudiés. La résistance induite (par divers agents) se traduit généralement par une réduction de la taille et/ou du nombre de lésions locales produites (LOEBENSTEIN, 1972 ; HAMILTON, 1980 ; SeIla, 1982). Cependant, certains traitements (incubation préalable à l'obscurité, éthylène) peuvent augmenter le nombre de lésions locales produites (LOEBENSTEIN, 1972). Pourtant, l'éthylène est considéré comme un stimulateur des réactions de défense des plantes vis-àvis de parasites fongiques (ESQUERRE-TUGAYE, 1977 ; HENFLING et al., 1978).

Dans le même sens, FrASER (1981) a montré qu'une infection localisée préalable, normalement inductrice de résistance, pouvait dans certaines conditions augmenter le nombre de lésions locales produites par une $2^{\mathrm{e}}$ inoculation virale.

Les 2 fractions G4 et G5 sont assez différentes l'une de l'autre, à la fois par leurs propriétés physicochimiques et leur activité élicitrice. Selon toute vraisemblance, la fraction $\mathrm{G} 4$, insoluble dans l'eau, pourrait être un composé lipidique (un glycéride) assez proche des substances mises en évidence chez Phytophthora infestans (BOSTOCK et al., 1982). En revanche, G5, hydrosoluble, s'apparenterait aux glucomannanes isolées de Phytophthora megasperma $f$. $s p$. glycinea par une endoglucanase $\beta 1-3$ élaborée par le soja (KEEN et al., 1983). Jusqu'à plus ample information, il ne semble pas que $P$. capsici produise des polymères d'hexosamines de type « chitin ou chitosan " (HADWIGER \& LOSCHKE, 1981).

L'obtention de réactions de défense plus importantes chez les jeunes plantes que chez les organes en survie ainsi que la persistance temporaire d'effets physiologiques polyvalents laissent entrevoir des possibilités d'application pratique. Beaucoup de plantes semblent posséder des potentialités de résistance avec conditionnement des cellules à distance, de telle sorte que les mécanismes de défense se mettent en place plus rapidement et plus intensément lors d'une infection (TOUZÉ \& ROSSIGNOL, 1980 ; KUĆ, 1982).

A l'issue de cette étude, un certain nombre de points restent à élucider. En particulier, des essais préliminaires non relatés ici ont montré qu'après pulvérisation de la fraction G5 sur l'ensemble du feuillage intact, on n'observe aucun effet protecteur vis-à-vis d'un champignon parasite appliqué $48 \mathrm{~h}$ après dans 
les mêmes conditions ; pourtant élicitation et contamination sont alors réalisées sur les mêmes sites. Cet échec pourrait provenir d'un mauvais contrôle de la pression d'inoculum ou d'une concentration trop faible en éliciteur à la surface du végétal.

Reçu le 6 juillet 1983.
Accepté le 14 mai 1984.

\section{REMERCIEMENTS}

Les auteurs remercient vivement S. GEBRE et J. P. Leroux pour leur contribution aux infections virales et au dénombrement des lésions locales.

\section{RÉFÉRENCES BIBLIOGRAPHIQUES}

Anderson-Prouty A., Albersheim P., 1975. Host-pathogen interactions. VIII. Isolation of a pathogen synthesized fraction rich in glucan that elicits a defence response in the pathogen's host. Plant Physiol., 56, 286-291.

Ayers A. R., Valent B., Ebel J., Albersheim P., 1976. Hostpathogen interactions. XI. Composition and structure of wallreleased elicitor fractions. Plant Physiol., 57, 766-774.

Bostock R. M., Laine R. A., Kuć J. A., 1982. Factors affecting the elicitation of sesquiterpenoid phytoalexin accumulation by eicosapentaenoic and arachidonic acids in potato. Plant Physiol., 70, 1417-1424.

Cline K., Wade M., Albersheim P., 1978. Host-pathogen interactions. XV. Fungal glucans which elicit phytoalexin accumulation in soybean also elicit the accumulation of phytoalexins in other plants. Plant Physiol., 62, 918-921.

Coulomb Ph. J., Molot P. M., Mas P., Conus M., Ferrière H., $1980 a$. Mise en évidence d'une induction de capsidiol et de résistance chez Capsicum annuum par certaines fractions cellulaires obtenues après fractionnement du mycélium de Phytophthora capsici. C. R. Acad. Sci. Paris, t. 290, Sér. D, 275-277.

Coulomb Ph. J., Molot P. M., Coulomb C., Mas P., 1980b. Etude d'une induction de résistance chez les cotylédons de Capsicum annuum par des fractionnements membranaires du mycélium de Phytophthora capsici à l'aide d'un nouveau test biologique. Ann. Phytopathol., 12 (4), 389-394.

De Wit P. J. G. M., Roseboom P. H. M., 1980. Isolation, partial characterization and specificity of glycoprotein elicitors from culture filtrates, mycelium and cell walls of Cladosporium fulvum. Physiol. Plant Pathol., 16, 391-408.

De Wit P. J. G. M., Spikman G., 1982. Evidence for the occurrence of race and cultivar specific elicitors of necrosis in intercellular fluids of compatible interaction of Cladosporium fulvum and tomato. Physiol. Plant Pathol., 21, 1-11.

Doke N. N., Garas N. A., Kuć J., 1979. Partial characterization and aspects of the mode of action of hypersensitivity-inhibiting factor (H.I.F.) isolated from Phytophthora infestans. Physiol. Plant Pathol., 15 (2), 127-140.

Esquerré-Tugaye M. T., 1977. Les glycoprotéines des surfaces cellulaires végétales : étude particulière d'une glycoprotéine à hydroxyproline dans les plantes de melon au cours d'une maladie parasitaire. Thèse Doct. Sci. Biol., Toulouse, 144 p.

Fraser R. S. S., 1981. Are the "pathogenesis-related" proteins involved in acquired systemic resistance of tobacco plants to tobacco mosaic virus ? J. Gen. Virol., 58, 305-313.

Garas N. A., Kuć J., 1981. Potato lectin lyses zoospores of Phytophthora infestans and precipitates elicitors of terpenoid accumulation produced by the fungus. Physiol. Plant Pathol., 18, 227 237.

Gianinazzi S., 1982. Antiviral agents and inducers of virus resistance : analogies with interferon. In R. K. S. Wood : «Active defence mechanism in plants ", Plenum Press, N. Y.-London, p. 275-298.

Gianinazxi S., Martin C., 1975. A natural compound inducing resistance to virus infection in plants. Phytopathol. Z., 83 (1), 23-26.

Gonzales-Garza R., Gumpf D. J., Kishaba A. N., Bohn G. W., 1979. Identification, seed transmission and host range pathogenicity of a California isolate of Melon Necrotic Spot Virus. Phytopatho$\log y, 69,340-345$.
Green T. R., Ryan C. A., 1973. Wound-induced proteinase inhibitor in tomato leaves. Some effects of light and temperature on the wound response. Plant Physiol., 51, 19-21.

Hadwiger L. A., Loschke D. C., 1981. Molecular communication in host-parasite interactions : hexosamine polymers (chitosan) as regulator compounds in race-specific and other interactions. Phytopathology, 71, 755-761.

Hamilton R. I., 1980. Defences triggered by previous invaders : Viruses. In Horsfall \& Cowling : « Plant Disease », vol. 5, Academic Press, 279-303.

Henfling J. W. D. M., Lisker N., Kuć J., 1978. Effect of ethylene on phytuberin and phytuberol accumulation in potato tuber slices. Phytopathology, 68, 857-862.

Jenns A. E., Caruso F. L., Kuć J., 1979. Non-specific resistance to pathogens induced systemically by local infection of cucumber with tobacco necrosis virus, Colletotrichum lagenarium or Pseudomonas lachrymans. Phytopathol. mediter., 18, 129-134.

Keen N. T., Yoshikawa M., Wang M. C., 1983. Phytoalexin elicitor activity of carbohydrates from Phytophthora megasperma $f$. sp. glycinea and other sources. Plant Physiol., 71, 466-471.

Kuć J., 1982. Plant immunization-mechanisms and practical implications. In R. K. S. Wood: "Active defence mechanisms in plants », Plenum Press, New York, p. 157-178.

Loebenstein G., 1972. Localization and induced resistance in virus infected plants. Annu. Rev. Phytopathol., 10, 177-206.

Marchoux G., 1975. Propriétés biologiques et génétiques des ARN du virus de la mosaïque du concombre. Thèse Doct. Sc. Nat., CNRS A0 11489.

Marrou J., 1967. Amélioration des méthodes de transmission mécanique des virus par adsorption des inhibiteurs d'infection sur le charbon végétal. $C$. R. Acad. Agric. Fr., 53, 972-981.

Marrou J., Risser G., 1967. La criblure du melon. Etude préliminaire d'une nouvelle maladie à virus du melon en culture sous serre. Ann. Epiphyt., 18, $\mathrm{n}^{\circ}$ HS « Etudes de Virologie », 193-203.

Molot P. M., Mas P., Conus M., Ferrière H., 1980a. La résistance du piment à Phytophthora capsici. VII. Protection des organes foliaires après mise en survie sur un filtrat de culture du parasite. Caractérisation des conditions d'expression du phénomène. Ann. Phytopathol., 12 (2), 95-107.

Molot P. M., Staron T., Mas P., 1980b. La résistance du piment à Phytophthora capsici. VIII. Induction de résistance et de capsidiol chez Capsicum annuum avec des fractions obtenues à partir des filtrats de culture et du mycélium de Phytophthora capsici. Ann. Phytopathol., 12 (4), 379-387.

Molot P. M., Pochard E., Mas P., 1983. La résistance du piment (Capsicum annuum) à Phytophthora capsici. XI. Réponse de 5 lignées de piment à une «fraction élicitrice »; influence de la dose d'éliciteur et efficacité de la protection induite vis-à-vis de plusieurs souches du parasite. Agronomie, 3 (4), 327-332.

Roberts D. A., 1983. Acquired resistance to Tobacco Mosaic Virus transmitted to the progeny of hypersensitive tobacco. Virology, 124, 161-163.

Roby D., 1981. Induction par des éliciteurs fongiques via l'éthylène, de réactions associées à la défense des plantes. Thèse Doct. $3^{\mathrm{e}} \mathrm{cycle}$, Univ. Toulouse, $67 \mathrm{p}$.

Rossignol M., 1979. La prémunition des plantes de melon contre l'anthracnose. Thèse Doct. spécialité, Univ. Toulouse, $63 \mathrm{p}$. 
Ryan C. A., 1978. Proteinase inhibitors in plant leaves : a biochemical model for pest-induced natural plant protection. TIBS, 3, 7, juillet $78,148-150$.

Seila I., 1981. Plant virus interactions related to resistance and localization of viral infections. $A d v$. Virus Res., 26, 201-237.

Staub T. H., Kuć J., 1980. Systemic protection of cucumber plants against disease caused by Cladosporium cucumerinum and Colleto- trichum lagenarium by prior infection with either fungus. Physiol. Plant Pathol., 17, 389-393.

Touzé A., Rossignol M., 1980. La protection biologique des plantes contre des infections bactériennes et fongiques. Ann. Phytopathol., 12 (4), 457-465

Troutman J. L., Fulton R. W., 1958. Resistance in tobacco to cucumber mosaic virus. Virology, 6, 303-316. 